\title{
1-P-098 Poster Sessions
}

\section{SIRT1 activitors ameliorate the ability of cell membrane repair.}

\author{
Azekami Kuya, Atsushi Kuno, Ryusuke Hosoda, Yoshiyuki Horio
}

Dept. Pharmacol., Sapporo Med. Univ.,Sch.Med.

Background:It is known that there is a function of cell survival such as oxidant stress resistance and NAD + dependent histone deacetylation by SIRT1 activitots. We previously reported that administration of activator of SIRT1, resveratorol (Rsv), ameliorates muscular pathophysiology of dystrophin-deficient mdx mice, and inhibition of SIRT1 suppresses membrane repair process of $\mathrm{C} 2 \mathrm{C} 12$ myoblasts and myotubes. However, it remains uncrear whether cell membrane repair in muscle cells is accelerated by SIRT1 activators. The purpose of this study is to prove that SIRT1 activators improve cell membrane repair ability as a factor in cell survival.

Method:In the present study, we used C2C12 myoblasts and myotubes. After treating these with Rsv (50 $\mu \mathrm{M})$ and nicotinamide mononucleotide (NMN) $(5 \mathrm{mM})(24 \mathrm{~h})$, we damaged the cell surface by local laser irradiation using a confocal microscope (Nikon A1). And membrane resealing process was monitored by an influx of fluorescent dye FM1-43 in cells surface after laser irradiation.

Result:We found that levels of fluorescence FM1-43 dye intake after laser-induced membrane destruction in C2C12 cells were significantly decreased in NMN (54.9 $\pm 18.9 \%)$ and Rsv (48.0 $\pm 20.4 \%)$ respectively.

Conclusion:Our data indicate a possibility that SIRT1 activators ameliorate muscular pathophysiology of muscular dystrophies through promoting plasma membrane repair. 